Research Paper

\title{
Malignant Melanoma in the Elderly: Diiferent Regional Disease and Poorer Prognosis
}

\author{
James B. Macdonald ${ }^{1}$, Amylou C. Dueck², Richard J. Gray³, Nabil Wasif ${ }^{3}$, David L. Swanson ${ }^{1}$, Aleksandar \\ Sekulic ${ }^{1}$, Barbara A. Pockaj ${ }^{3}$
}

1. Department of Dermatology, Mayo Clinic Arizona, 5777 E Mayo Boulevard, Phoenix, AZ 85054, USA.

2. Department of Biostatistics, Mayo Clinic Arizona, 5777 E Mayo Boulevard, Phoenix, AZ 85054, USA.

3. Department of Surgery, Mayo Clinic Arizona, 5777 E Mayo Boulevard, Phoenix, AZ 85054, USA.

$\triangle$ Corresponding author: Barbara A. Pockaj, M.D. Mayo Clinic Arizona, 5777 E Mayo Boulevard, Phoenix, AZ 85054 Phone: (480) 342-2000 Fax: (480) 342-2866 pockaj.barbara@mayo.edu

(C) Ivyspring International Publisher. This is an open-access article distributed under the terms of the Creative Commons License (http://creativecommons.org/ licenses/by-nc-nd/3.0/). Reproduction is permitted for personal, noncommercial use, provided that the article is in whole, unmodified, and properly cited.

Received: 2011.08.24; Accepted: 2011.10.20; Published: 2011.11.01

\begin{abstract}
Purpose: Age is a poor prognostic factor in melanoma patients. Elderly melanoma patients have a different presentation and clinical course than younger patients. We evaluated the impact of age $\geq 70$ years ( $y r s$ ) on the diagnosis and natural history of melanoma.

Methods: Retrospective review of 610 patients with malignant melanoma entered into a prospective sentinel lymph node (SLN) database, treated from June 1997 to June 2010. Disease characteristics and clinical outcomes were compared between patients $\geq 70$ yrs vs. $<70$ yrs of age.

Results: 237 patients (39\%) were $\geq 70$ yrs. Elderly patients had a higher proportion of head and neck melanomas ( $34 \%$ vs. $20 \%, p<0.00 \mathrm{I})$, and greater mean tumor thickness $(2.4 \mathrm{~mm}$ vs. $1.8 \mathrm{~mm}, \mathrm{p}<0.00 \mathrm{I})$. A greater proportion of T3 or T4 melanoma was seen in the elderly $(p<0.00 \mathrm{I})$ as well as a greater mean number of mitotic figures: $3.6 / \mathrm{mm}^{2} \mathrm{vs} .2 .7 / \mathrm{mm}^{2}(p=0.005)$. Despite greater mean thickness, the incidence of SLN metastases was less in the $\geq 70$ yrs group with T3/T4 melanomas ( $8 \%$ vs. $33 \%, p=0.02)$. The elderly had a higher rate of local and in-transit recurrences, $14.5 \%$ vs. $3.4 \%$ at 5 yrs $(p<0.00 \mathrm{I}) .5 \mathrm{yr}$ disease-specific mortality and overall mortality were worse for those $\geq 70$ yrs: $16 \%$ vs. $8 \%(p=0.004)$, and $30 \%$ vs. $12 \%$ $(p<0.00 \mathrm{I})$, respectively.

Conclusions: Elderly ( $\geq 70 \mathrm{yrs}$ ) melanoma patients present with thicker melanomas and a higher mitotic rate but have fewer SLN metastases. Melanoma in the elderly is more common on the head and neck. Higher incidence of local/in-transit metastases is seen among the elderly. Five-year disease-specific mortality and overall mortality are both worse for these patients.
\end{abstract}

Key words: Elderly patients, metastases, melanoma

\section{Introduction}

The incidence of malignant melanoma has been increasing over the last several decades. Melanoma is the sixth most common malignancy in the United States; there were an estimated 68,120 new cases of invasive melanoma in 2010 with 8,700 deaths due to the disease 1, 2. When compared to younger age groups, mortality is significantly higher in the elderly implicating age as a poor prognostic factor ${ }^{3}$. As the 
proportion of the elderly among the world population continues to grow $^{3}$, nearing $20 \%$ in developed countries $^{4}$, so will the importance of melanoma characterization and proper risk stratification in that age group.

The clinical presentation and pathological characteristics in melanoma of the elderly differ from that of their younger counterparts. Melanoma of the elderly has been shown to be more common in men and to be discovered later in its development. The incidence of ulceration, high mitotic index, and advanced Breslow thickness have been shown to be higher in older age groups ${ }^{5-8}$. Paradoxically, despite lower overall survival in elderly with malignant melanoma, the rate of positive sentinel lymph nodes (SLN) decreases with age ${ }^{3,9-11}$. This observation remains unexplained but is likely related at least in part to atrophy of skin lymphatics that occur with age ${ }^{12}$.

Given the differing clinical presentation, pathological characteristics, and heightened mortality rates of elderly melanoma patients, we aimed to compare disease characteristics and clinical outcomes of patients 70 years of age and older versus under 70 years old.

\section{Methods}

We reviewed 610 patients with malignant melanoma requiring SLN biopsy entered into a prospective SLN database from Mayo Clinic Arizona, treated from June 1997 to June 2010. Approval was obtained from the Institutional Review Board. Disease characteristics and clinical outcomes were compared between patients $\geq 70$ yrs vs. $<70$ yrs of age. Subset analysis was performed with a breakdown between age groups of $<50,50-59,60-69$ and $>70$, but the statistical power of this analysis was limited due to the smaller number of events in the groups. Primary melanoma anatomic locations were divided in four locations: head and neck, lower extremity, trunk, and upper extremity. Characteristics of the primary melanoma that were analyzed included: Breslow's depth, T-stage $(<1 \mathrm{~mm}, 1-2 \mathrm{~mm}, 2.01-4 \mathrm{~mm},>4 \mathrm{~mm})$, ulceration, tumor infiltrating lymphocytes (TIL), preexisting nevus, angiolymphatic invasion, perineural invasion, and mitotic rate. Regional disease was assessed by SLN biopsy results. Disease outcome measures included type of recurrence (local/in-transit, regional, systemic), time to recurrence, time to local/in-transit recurrence, disease-specific mortality, and overall mortality.

Categorical variables were compared between groups using chi-squared tests. Continuous variables were compared between groups using two-sample independent samples t-tests. Disease outcomes were plotted using Kaplan-Meier curves and compared between groups using Cox proportional hazards models. Statistical software used included SAS software, version 9 (SAS Institute). P-values $<0.05$ were considered statistically significant throughout.

\section{Results}

$237(39 \%)$ patients with melanoma requiring SLN biopsy were $\geq 70$ years. The mean age in the $<70$ years old group was 52.2 (14-69). Among the elderly group ( $\geq 70 \mathrm{yrs})$, the mean age was 77.3 (70-97), there was a greater predominance of men, 153 (65\%) compare to those $<70 \mathrm{yrs}$, where men comprised $56 \%$ $(p=0.04)$, Table 1 . The location of melanomas was significantly different between the elderly and younger patients $(p<0.001)$. The elderly patients had a higher proportion of head and neck melanomas $(34 \%)$ relative to the younger patients $(19 \%)$, whose most prevalent site was the trunk (35\%).

Comparison of disease characteristics between age groups appears in Table 1 .

Table I: Patient and Melanoma Characteristics by Age Group.

\begin{tabular}{|c|c|c|c|}
\hline $\begin{array}{l}\text { Primary tumor charac- } \\
\text { teristics }\end{array}$ & $<70 \mathrm{yrs}$ & $\geq 70 \mathrm{yrs}$ & Total \\
\hline Male & $56 \%$ & $65 \%$ & $59 \%$ \\
\hline $\begin{array}{l}\text { Location } \\
\text { Head and Neck } \\
\text { Trunk } \\
\text { Upper Extremity } \\
\text { Lower Extremity }\end{array}$ & $\begin{array}{l}20 \% \\
35 \% \\
21 \% \\
24 \%\end{array}$ & $\begin{array}{l}34 \% \\
23 \% \\
22 \% \\
22 \%\end{array}$ & $\begin{array}{l}25 \% \\
30 \% \\
21 \% \\
23 \%\end{array}$ \\
\hline Pre-existing Nevus & $36 \%$ & $17 \%$ & $29 \%$ \\
\hline $\begin{array}{l}\text { Mean Breslow Level } \\
\text { (SD) }\end{array}$ & $1.8(1.5)$ & $2.4(2.5)$ & $2.0(1.9)$ \\
\hline $\begin{array}{l}\text { T Stage } \\
\text { T1 } \\
\text { T2 } \\
\text { T3 } \\
\text { T4 }\end{array}$ & $\begin{array}{l}33 \% \\
43 \% \\
15 \% \\
8 \%\end{array}$ & $\begin{array}{l}19 \% \\
39 \% \\
31 \% \\
12 \%\end{array}$ & $\begin{array}{l}28 \% \\
41 \% \\
21 \% \\
9 \%\end{array}$ \\
\hline $\begin{array}{l}\text { Tumor Infiltrating } \\
\text { Lymphocytes } \\
\text { Absent } \\
\text { Non-brisk } \\
\text { Brisk }\end{array}$ & $\begin{array}{l}34 \% \\
46 \% \\
21 \%\end{array}$ & $\begin{array}{l}32 \% \\
50 \% \\
18 \%\end{array}$ & $\begin{array}{l}33 \% \\
47 \% \\
20 \%\end{array}$ \\
\hline Ulceration & $23 \%$ & $29 \%$ & $26 \%$ \\
\hline $\begin{array}{l}\text { Angiolymphatic or Per- } \\
\text { ineural Invasion }\end{array}$ & $11 \%$ & $15 \%$ & $13 \%$ \\
\hline $\begin{array}{l}\text { Mean Mitotic Figure } \\
\text { Rate [per } \mathrm{mm}^{2} \text { ] (SD) }\end{array}$ & $2.7(3.0)$ & $3.6(4.2)$ & $3.0(3.5)$ \\
\hline $\begin{array}{l}\text { SLN+ } \\
\text { Entire cohort } \\
\text { T1/2 } \\
\text { T3/4 }\end{array}$ & $\begin{array}{l}15 \% \\
9 \% \\
33 \%\end{array}$ & $\begin{array}{l}11 \% \\
6 \% \\
18 \%\end{array}$ & $13 \%$ \\
\hline
\end{tabular}

$\mathrm{SD}=$ standard deviation

Elderly patients had thicker tumors, with $43 \%$ of elderly patients had a T3 or T4 melanoma compared to $23 \%$ in the non-elderly $(\mathrm{p}<0.001)$. Patients with age 
$\geq 70$ had a greater mean number of mitotic figures, $3.6 / \mathrm{mm}^{2}$ vs. $2.7 / \mathrm{mm}^{2}(\mathrm{p}=0.005)$ including a greater preponderance of lesions with a high mitotic rate $>5 / \mathrm{mm}^{2}(29 \%$ vs $17 \%, \mathrm{p}=0.003$. The elderly patients did not have a greater prevalence of ulceration. Tumors in the elderly were less likely to arise from a pre-existing nevus, $17 \%$ vs. $36 \%$ ( $p=0.003$ ). There were no differences in tumor infiltrating lymphocyte status or angiolymphatic invasion.

Overall, the prevalence of SLN metastases was similar between elderly and non-elderly patients. When the incidence of SLN metastases was stratified by $\mathrm{T}$ stage (T1/T2 and $\mathrm{T} 3 / \mathrm{T} 4)$, the rate of positive SLNs was dramatically less common in elderly patients with T3 or T4 melanoma versus their younger counterparts ( $18 \%$ vs. $33 \%, p=0.02)$.

The median follow up time was 3.8 years (range $0-12.6$ years). The elderly patients had a higher rate of recurrence (including local/in-transit, regional, or systemic recurrence) relative to the younger patients ( $25.2 \%$ vs $9.1 \% 5$ years after surgery; hazard ratio $[\mathrm{HR}]=2.6, \mathrm{p}<0.001$; Figure 1A). Additionally, the elderly patients had a higher rate of local/in-transit recurrence $(14.5 \%$ vs $3.4 \% 5$ years after surgery; $\mathrm{HR}=3.6, \mathrm{p}<0.001$; Figure $1 \mathrm{~B}$ ) and systemic recurrence $(14.9 \%$ vs $6 \% 5$ years after surgery; $\mathrm{HR}=1.83, \mathrm{p}=0.05$; figure 1C).

Subset analysis of age groups <50, 50-59, 60-69 and $>70$ showed progressively worse outcomes with age, as expected. Recurrence-free survival rates in the subset analysis were $91.8 \%, 85.6 \%, 82 \%$, and $60 \%$ respectively for age groups in ascending order. Relative to age group $<50$, hazard ratios for the age groups $50-59,60-69$, and $>70$ were $1.98(\mathrm{p}=0.08), 2.15(\mathrm{p}=0.03)$, and $5.42(\mathrm{p}<0.001)$ respectively.

5 -year mortality rate from all causes was greater in the elderly group, $29.8 \%$ vs. $12.3 \% 5$ years after surgery ( $\mathrm{HR}=3.0, \mathrm{p}<0.001$; Figure $2 \mathrm{~A})$. Additionally, 5 -year disease-specific mortality was higher for those $\geq 70$ years old: $16.1 \%$ vs. $8.2 \%$ ( $\mathrm{HR}=2.3, \mathrm{p}=0.004$; Figure 2B). In a multivariate model of melanoma-specific survival, age remained significant after adjusting for known prognostic factors of tumor thickness and positive SLN status.

\section{Discussion}

In our cohort of patients undergoing SLN biopsy, melanoma in the elderly was more common on the head and neck, less likely to arise from a pre-existing nevus, had greater mean tumor thickness and more mitotic figures than melanoma in their younger counterparts. Additionally, higher T-stage melanoma in the elderly was less likely to result in positive sentinel node biopsy. Furthermore, elderly melanoma patients had worse outcomes. They were more likely to have recurrence, including a higher rate of lo$\mathrm{cal} /$ in-transit recurrence and systemic recurrence. Consequently, they had a higher disease-specific mortality and worse overall survival.
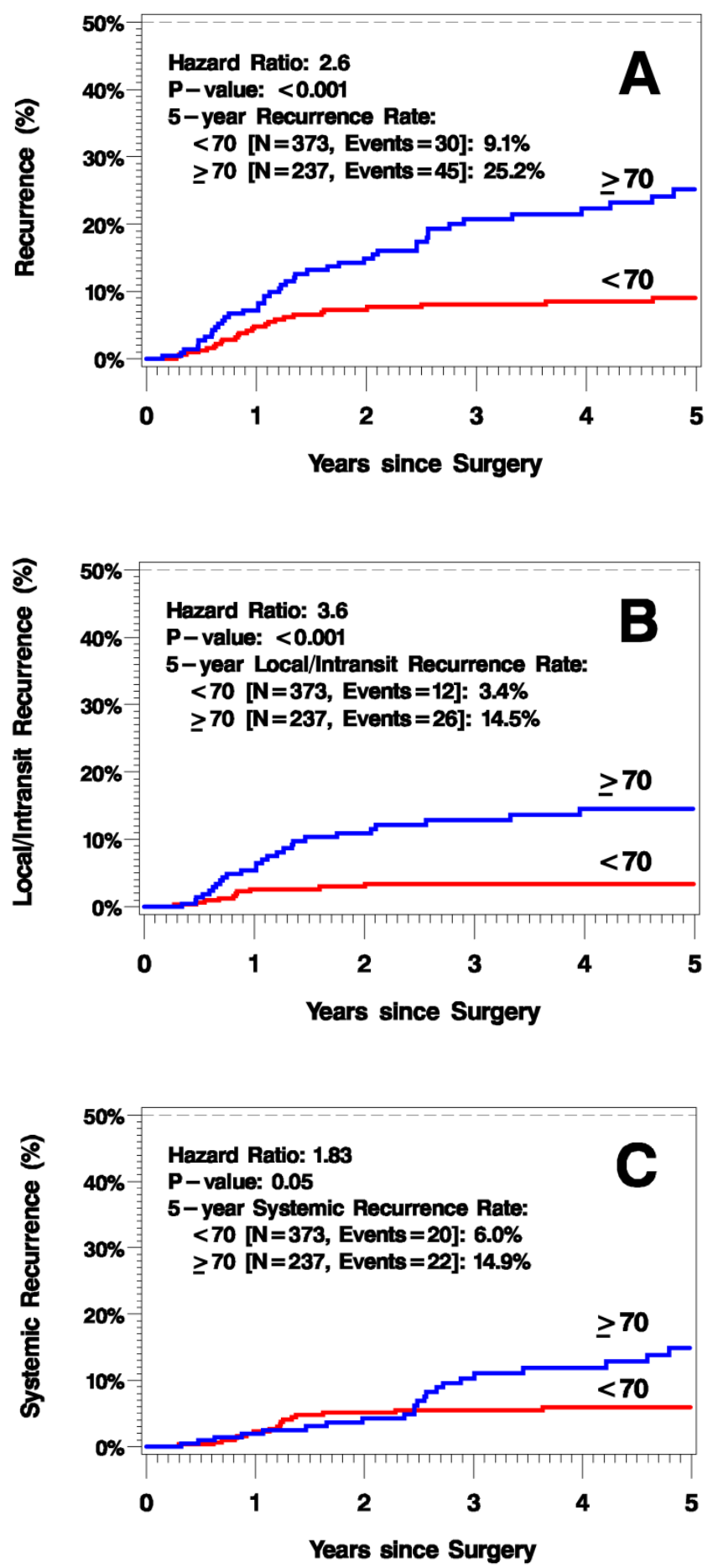

Figure I: Kaplan-Meier Plots of Time to Recurrence (A), Time to Local/In-Transit Recurrence (B), and Time to Systemic Recurrence (C). 

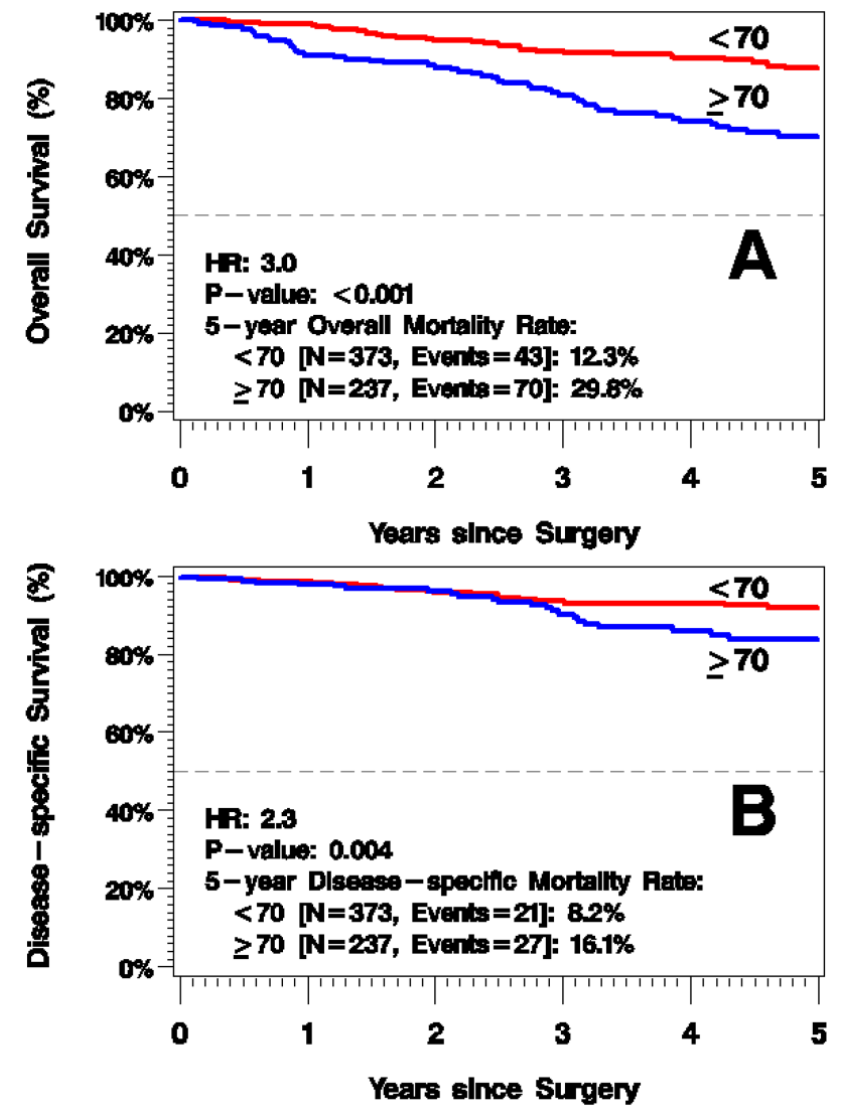

Figure 2: Kaplan-Meier Plots of Overall Survival (A) and Disease-Specific Survival (B).

Older age is recognized as an independent poor prognostic factor in melanoma patients. Caraco et al. studied 399 patients with melanoma who underwent SLN biopsy and compared patients $\leq 50$ years vs. $>50$ years. They found the melanoma patients $>50$ to have thicker lesions and a higher incidence of ulceration but no difference in the incidence of lymph node metastases. Overall survival was worse among the group $>50$ and age was a significant prognostic variable in multivariate analysis ${ }^{13}$. Data from the SEER database and Medicare enrollment and claims files, showed that increasing age after 65 years and melanoma detection by a dermatologist were significantly predictive of survival on multivariate analysis in a population of over 2000 patients ${ }^{14}$. In a large dataset of 17,600 melanoma patients, age was an independent prognostic factor for overall survival which was consistent within each thickness subgroup ${ }^{9}$. Worse prognosis in the elderly may be attributable to the fact that the elderly, especially elderly men, melanoma has been shown to have a more rapid growth rate ${ }^{15}$, thicker tumors ${ }^{16}$ and histologic ulceration ${ }^{16}$. Age as a poor prognostic factor may be the result of weakened immune defense mechanisms 17,18 , which may play a role in melanoma progression. Lastly, competing co-morbidities are much more prevalent in older patients, further contributing to higher overall mortality.

Elderly melanoma patients have been shown to present with tumors of advanced Breslow level 3, 9, 19, 20. Late diagnosis has been implicated as a cause for thicker melanoma with poor prognosis in the elderly. Multiple factors are likely involved in late discovery of cutaneous malignancies in the elderly. Some reasons may include scarcely visible anatomical locations of new pigmented lesions (i.e. scalp, back), loss of a partner's input on home examination, poor vision, less importance placed on changing lesions, and confusing new or changing benign seborrheic keratoses for pigmented lesions ${ }^{21,22}$. Furthermore, early detection programs for melanoma have generally targeted younger age groups. Studies have shown disproportionately low screening of elderly men despite this population accounting for $44 \%$ of melanoma cases discovered ${ }^{23}$.

Higher incidence of head and neck melanoma is likely attributable to cumulative photodamage and higher incidence of lentigo maligna melanoma as patients age. Swetter et al. showed that lentigo maligna melanoma was the only melanoma subtype with increasing incidence over a ten-year period and was more common in men $\geq 65$ years ${ }^{24}$. As the percentage of elderly persons in the world's population continues to grow, physicians performing melanoma screening should devote heightened attention to heavily sun-exposed sites where melanoma is more and more commonly discovered.

Our data confirmed previously reported findings that the elderly are less likely to have melanoma associated with a pre-existing nevus ${ }^{25}$. Malignant progression may be the result of continuous and cumulative photodamage to melanocytes not within a nevus ${ }^{26}$. This has clinical implications to patient education and early detection of melanoma in that age group. When counseling elderly patients regarding self-screening, a shift of focus from characteristics of changing nevi (ABCDE of melanoma) to awareness of any new growths may lead to more routine screening and earlier detection.

Our data demonstrated that the mitotic rate in the elderly melanoma patients was higher than their younger counterparts. Mitotic rate has been associated with worse clinical outcomes in melanoma patients and has been identified as an independent prognostic factor in multiple studies ${ }^{27-29}$. In a multifactorial analysis of 10,233 patients with clinically localized melanoma, mitotic rate was the second best prognostic factor of survival behind tumor thickness ${ }^{29}$. In a 
series of more than 1200 cases, mitotic rate was significantly associated with survival and more prognostic than tumor ulceration ${ }^{28}$. Higher mitotic rate tumor burden has also been implicated as a factor contributing to a greater likelihood of local/in-transit recurrence and poor clinical outcomes ${ }^{30}$. Paek et al. showed mitotic rate is associated with positive SLN, but the impact of mitotic activity on SLN result was less in the elderly compared to younger cohorts ${ }^{31}$. Our data was consistent with these observations as our elderly population had more mitotic figures, greater number of non-regional recurrences, and worse clinical outcomes. Additionally, they had a lower positive SLN rate, indicating that mitotic activity had less impact on SLN result in the elderly compared to younger patients.

In advanced T-stage melanoma, despite worse disease-specific and overall mortality, the elderly had far fewer cases of positive SLN biopsies. This paradox is consistent with evidence from prior studies 3, 9-11. Chao et al. studied 5 age groups of melanoma patients in a large series of over 3000 patients. They showed a progressive decline in incidence of SLN metastasis with increasing age across all five age groups. The oldest group ( $>60$ years) had a positive SLN rate of $14.4 \%$, significantly less than the next nearest age groups ${ }^{3}$. Our cohort of elderly patients ( $\geq 70$ years) demonstrated a comparable rate of positive SLN in $11 \%$.

The reason for fewer cases of positive sentinel lymph node biopsies remains uncertain but may be accounted by altered lymphatic drainage in the elderly ${ }^{12}$. Although the mode of spread in any cancer is never certain, it appears that like younger patients, melanoma in the elderly principally spreads along lymphatic routes as there are more in-transit metastases noted in this age group ${ }^{32}$. UV irradiation has been shown to induce VEGF-A-mediated damage of cutaneous lymphatic channels 33,34 . Lifelong accumulation of photodamage is likely contributory to disruption of lymphatic channels, as well as gravity-dependent degeneration, disallowing complete migration of tumor cells to the sentinel node in areas of atrophied lymphatic channels in the elderly.

Older age in this study was associated with shorter time to recurrence. Time to recurrence has been shown to be an independent predictor of post-recurrence survival ${ }^{35}$. Additionally, advanced age at the time of recurrence diagnosis has been associated with poor prognosis ${ }^{36-38}$. Murali et al. studied 279 melanoma patients with recurrence and found that those patients who had recurrence in 12 months or less from treatment of primary melanoma had significantly worse post-recurrence survival and pro- gression-free survival rates ${ }^{35}$. A recent long-term follow-up study of 2487 patients showed that advanced age, male sex, high $\mathrm{pN}$, and distant metastases as the first site of recurrence were associated with worse survival outcomes after the manifestation of recurrent disease ${ }^{38}$.

This study has limitations. The analysis relied on data obtained from a single institution. Subset analysis of age groups $<50,50-59,60-69,>70$ was of limited statistical power because of small number of events in each group. Data was retrieved only from patients undergoing sentinel lymph node biopsy; given the potential selection bias, it may not be valid to generalize conclusions to the entire elderly population with melanoma. Lastly, the follow-up period is limited to a mean of 3.8 years, which may influence outcome data to reflect more poorly on the elderly population.

Given the differing clinical presentation, primary melanoma characteristics, and disparate disease-specific outcomes of melanoma in the elderly compared to non-elderly patients, clinicians and surgeons should be mindful of proper screening and risk-stratification of elderly patients when participating in their care. Due to the higher risk of in-transit disease, follow-up should focus on loco-regional recurrences. These clinical differences may have significant implications for clinical trials. Many adjuvant clinical trials are geared to patients with lymph node positive disease. Due to the fact that the elderly have a much lower incidence of lymph node positive disease but otherwise poorer prognosis, age may need to be included when developing clinical trials in order to evaluate the efficacy of adjuvant therapy in this patient population.

\section{Conflict of Interest}

The authors have declared that no conflict of interest exists.

\section{References}

1. Jemal A, Siegel R, Xu J, Ward E. Cancer statistics, 2010. CA Cancer J Clin. 2010;60: 277-300.

2. Lasithiotakis KG, Petrakis IE, Garbe C. Cutaneous melanoma in the elderly: epidemiology, prognosis and treatment. Melanoma Res. 2010;20: 163-170.

3. Chao C, Martin RC, 2nd, Ross MI, et al. Correlation between prognostic factors and increasing age in melanoma. Ann Surg Oncol. 2004;11: 259-264.

4. Rosenthal RA, Kavic SM. Assessment and management of the geriatric patient. Crit Care Med. 2004;32: S92-105.

5. Alexander HR, Turnbull AD, Salamone J, Keefe D, Melendez J. Upper abdominal cancer surgery in the very elderly. J Surg Oncol. 1991;47: 82-86.

6. MacKie RM. Malignant melanoma: clinical variants and prognostic indicators. Clin Exp Dermatol. 2000;25: 471-475.

7. MacKie RM, Hole D, Hunter JA, et al. Cutaneous malignant melanoma in Scotland: incidence, survival, and mortality, 
1979-94. The Scottish Melanoma Group. BMJ. 1997;315: 1117-1121.

8. Ramesh HS, Pope D, Gennari R, Audisio RA. Optimising surgical management of elderly cancer patients. World J Surg Oncol. 2005;3: 17.

9. Balch CM, Soong SJ, Gershenwald JE, et al. Prognostic factors analysis of 17,600 melanoma patients: validation of the American Joint Committee on Cancer melanoma staging system. J Clin Oncol. 2001;19: 3622-3634.

10. Jemal A, Siegel R, Ward E, et al. Cancer statistics, 2008. CA Cancer J Clin. 2008;58: 71-96.

11. Sondak VK, Taylor JM, Sabel MS, et al. Mitotic rate and younger age are predictors of sentinel lymph node positivity: lessons learned from the generation of a probabilistic model. Ann Surg Oncol. 2004;11: 247-258.

12. Conway WC, Faries MB, Nicholl MB, et al. Age-related lymphatic dysfunction in melanoma patients. Ann Surg Oncol. 2009;16: 1548-1552.

13. Caraco C, Marone U, Botti G, Celentano E, Lastoria S, Mozzillo $\mathrm{N}$. Age as predictor in patients with cutaneous melanoma submitted to sentinel lymph node biopsy. Eur J Surg Oncol. 2006;32: 970-973.

14. Pennie ML, Soon SL, Risser JB, Veledar E, Culler SD, Chen SC. Melanoma outcomes for Medicare patients: association of stage and survival with detection by a dermatologist vs a nondermatologist. Arch Dermatol. 2007;143: 488-494.

15. Liu W, Dowling JP, Murray WK, et al. Rate of growth in melanomas: characteristics and associations of rapidly growing melanomas. Arch Dermatol. 2006;142: 1551-1558.

16. Lasithiotakis $\mathrm{K}$, Leiter $\mathrm{U}$, Meier F, et al. Age and gender are significant independent predictors of survival in primary cutaneous melanoma. Cancer. 2008;112: 1795-1804.

17. Hegde UP, Chakraborty N, Kerr P, Grant-Kels JM. Melanoma in the elderly patient: relevance of the aging immune system. Clin Dermatol. 2009;27: 537-544.

18. Tsai S, Balch C, Lange J. Epidemiology and treatment of melanoma in elderly patients. Nat Rev Clin Oncol. 2010;7: 148-152.

19. Loggie B, Ronan SG, Bean J, Das Gupta TK. Invasive cutaneous melanoma in elderly patients. Arch Dermatol. 1991;127: 1188-1193.

20. Rivers JK, Kelly MC, Kopf AW, Welkovich B, Bart RS. Age and malignant melanoma: comparison of variables in different age-groups. J Am Acad Dermatol. 1989;21: 717-722.

21. Wiener DC, Argote-Greene LM, Ramesh H, Audisio RA, Jaklitsch MT. Choices in the management of asymptomatic lung nodules in the elderly. Surg Oncol. 2004;13: 239-248.

22. Adams J, Audisio RA, White M, Forman D. Age-related variations in progression of cancer at diagnosis and completeness of cancer registry data. Surg Oncol. 2004;13: 175-179.
23. Kelly JW. Melanoma in the elderly--a neglected public health challenge. Med J Aust. 1998;169: 403-404.

24. Swetter SM, Boldrick JC, Jung SY, Egbert BM, Harvell JD. Increasing incidence of lentigo maligna melanoma subtypes: northern California and national trends 1990-2000. J Invest Dermatol. 2005;125: 685-691.

25. Tsao H, Bevona C, Goggins W, Quinn T. The transformation rate of moles (melanocytic nevi) into cutaneous melanoma: a population-based estimate. Arch Dermatol. 2003;139: 282-288.

26. Testori A, Soteldo J, Sances D, et al. Cutaneous melanoma in the elderly. Melanoma Res. 2009;19: 125-134.

27. Azzola MF, Shaw HM, Thompson JF, et al. Tumor mitotic rate is a more powerful prognostic indicator than ulceration in patients with primary cutaneous melanoma: an analysis of 3661 patients from a single center. Cancer. 2003;97: 1488-1498.

28. Attis MG, Vollmer RT. Mitotic rate in melanoma: a reexamination. Am J Clin Pathol. 2007;127: 380-384.

29. Balch CM, Gershenwald JE, Soong SJ, et al. Final version of 2009 AJCC melanoma staging and classification. J Clin Oncol. 2009;27: 6199-6206.

30. Stucky CC, Gray RJ, Dueck AC, et al. Risk factors associated with local and in-transit recurrence of cutaneous melanoma. Am J Surg. 2010;200: 770-774.

31. Paek SC, Griffith KA, Johnson TM, et al. The impact of factors beyond Breslow depth on predicting sentinel lymph node positivity in melanoma. Cancer. 2007;109: 100-108.

32. Pawlik TM, Ross MI, Johnson MM, et al. Predictors and natural history of in-transit melanoma after sentinel lymphadenectomy. Ann Surg Oncol. 2005;12: 587-596.

33. Kajiya K, Hirakawa S, Detmar M. Vascular endothelial growth factor-A mediates ultraviolet B-induced impairment of lymphatic vessel function. Am J Pathol. 2006;169: 1496-1503.

34. Kajiya K, Sawane M, Huggenberger R, Detmar M. Activation of the VEGFR-3 pathway by VEGF-C attenuates UVB-induced edema formation and skin inflammation by promoting lymphangiogenesis. J Invest Dermatol. 2009;129: 1292-1298.

35. Murali R, Moncrieff MD, Hong J, et al. The prognostic value of tumor mitotic rate and other clinicopathologic factors in patients with locoregional recurrences of melanoma. Ann Surg Oncol. 2010;17: 2992-2999.

36. Francken AB, Accortt NA, Shaw HM, et al. Prognosis and determinants of outcome following locoregional or distant recurrence in patients with cutaneous melanoma. Ann Surg Oncol. 2008;15: 1476-1484

37. Reintgen DS, Cox C, Slingluff CL, Jr., Seigler HF. Recurrent malignant melanoma: the identification of prognostic factors to predict survival. Ann Plast Surg. 1992;28: 45-49.

38. Hohnheiser AM, Gefeller O, Gohl J, Schuler G, Hohenberger W, Merkel S. Malignant melanoma of the skin: long-term follow-up and time to first recurrence. World J Surg. 2010;35: 580-589. 